\title{
Conception et expérimentation de dispositifs microondes didactiques par impression 3D plastique
}

\author{
N. Jolly ${ }^{\mathrm{a}}$, O. Tantot ${ }^{\mathrm{a}}$, A. Périgaud ${ }^{\mathrm{a}}$, N. Delhote ${ }^{\mathrm{a}}$, \\ S. Bila ${ }^{\mathrm{a}}, \mathrm{S}$. Verdeyme $^{\mathrm{a}}, \mathrm{R}-\mathrm{Ph}$. Gramond $^{\mathrm{b}}$ \\ ${ }^{a}$ XLIM, UMR 7252, PLM pôle CNFM de Limoges, Université de Limoges, France \\ b Plateforme technologique RAMSEI'S, Lycée Turgot, Limoges, France \\ Contact email : olivier.tantot@unilim.fr
}

La conception de dispositifs passifs volumiques imprimés par fabrication additive 3D plastique pour des objectifs pédagogiques est présentée. Deux aspects sont exposés dans ce papier, le premier étant les dispositifs à réaliser dans le cadre de projet de master qui s'étalent sur plusieurs semaines avec une réalisation finale par impression plastique 3D. Le second étant l'utilisation de dispositifs déjà imprimés pour la compréhension physique du dispositif et de son réglage avec un appui logiciel lors de séance de travaux pratiques. L'impression 3D plastique permet, par le caractère faible coût des fabrications et de mise en œuvre aisée, des temps de fabrication de quelques heures. Des prototypes pour des projets de master microondes peuvent être ainsi envisagés. L'investissement des étudiants se faisant sur l'ensemble des phases d'ingénierie dans la philosophie "Do It Yourself". Les travaux pratiques deviennent plus ludiques grâce à des structures à assemblage et réglage rapides.

\section{Introduction}

Des études préliminaires ont démontré l'efficacité de la fabrication additive 3D plastique pour des solutions de prototypage rapide appliquées aux applications microondes $(1,2)$. Dans cet article, plusieurs structures réalisées par impression 3D plastique issues de projets sont présentées. Les premières structures ont été étudiées pour évaluer les performances de ces dispositifs microondes avec les dispersions dimensionnelles liées à cette technologie de fabrication et la limite fréquentielle supérieure qu'elles imposent. Dans cet objectif, plusieurs dispositifs avec une difficulté de conception croissante ont été réalisés lors de projets ou de stages de master de la spécialité «microondes ». Deux techniques d'impression 3D plastique ont été utilisées. La première est celle du dépôt de fil d'ABS ou de PLA fondu (Fused Deposition Modeling). Cette méthode est devenue très courante avec des imprimantes de faible coût d'achat et de consommable. La machine professionnelle utilisée est la Print SE de Stratasys avec des épaisseurs de couches de $0,254 \mathrm{~mm}$ et des précisions en XY inférieures à $200 \mu \mathrm{m}$ pour les pièces de grande taille (jusqu'à 150 x 150 x $200 \mathrm{~mm}$ ) et inférieures à $100 \mu \mathrm{m}$ pour les pièces de quelques centimètres (1). La deuxième imprimante (EDEN 260V) de marque Stratasys, utilise la technique de jet de gouttelettes de polymère durci par flash UV au fur et à mesure du dépôt (polyjet@). Suivant la rapidité d'impression, la couche de polymère est de 16 ou $33 \mu \mathrm{m}$ et la précision en XY est comprise entre 25 et $80 \mu \mathrm{m}$ pour des pièces inférieures à $50 \mathrm{~mm}$ et jusqu'à $200 \mu \mathrm{m}$ 
pour des pièces plus grandes (jusqu'à 225 x 252 x $200 \mathrm{~mm} 3$ ). Cette imprimante présente un cout de fabrication environ trois fois celui d'une imprimante de type FDM.

Deux dispositifs microondes fonctionnant à des fréquences jusqu'à $10 \mathrm{GHz}$ et imprimés en FDM sont présentés. La fin de la bande X semble être la limite fréquentielle atteignable avec les précisions de cette technique (3). L'impression par technique polyjet ${ }^{@}$ présente de meilleures précisions et des dispositifs fonctionnant jusqu'à $36 \mathrm{GHz}$ ont pu être mesurés avec des performances satisfaisantes (4). Un filtre volumique modulaire en bande $\mathrm{X}$ conçu pour être facilement et rapidement assemblé a été réalisé avec cette dernière imprimante. Les métallisations des faces intérieures et des brides de connexion ont été réalisées par une laque argent de marque Ferro. La conductivité de cette peinture est suffisamment importante pour assurer un comportement de plan conducteur $(\sigma \approx 1,5 \mathrm{~S} / \mu \mathrm{m})(4)$ pour le champ EM.

\section{Transition guide d'onde coaxial - guide d'onde rectangulaire}

Un premier projet a été initié pour connaitre la faisabilité de la technologie FDM ABS pour des dispositifs microondes volumiques passifs. Une fonction de transition de guide coaxial vers guide rectangulaire a été étudiée. Cette fonction est simple de conception et parfaitement adaptée à un projet de Master où les outils de simulations Electromagnétiques 3D (HFSS) sont abordés. L'optimisation automatique sous HFSS de la réponse en transmission s'est faite dans la bande de fréquence $6-8 \mathrm{GHz}$, bande choisie pour commencer à être sensible aux défauts dimensionnels des techniques de fabrication classiques pour les structures volumiques. La connectique coaxiale est un connecteur SMA avec une âme centrale entourée de Téflon qui plonge dans le guide rectangulaire (Fig1-a). L'optimisation a porté sur la forme d'un perturbateur à empilement au départ aléatoire de plaques métalliques en vis-à-vis de l'âme centrale (Fig. $1-b)$.
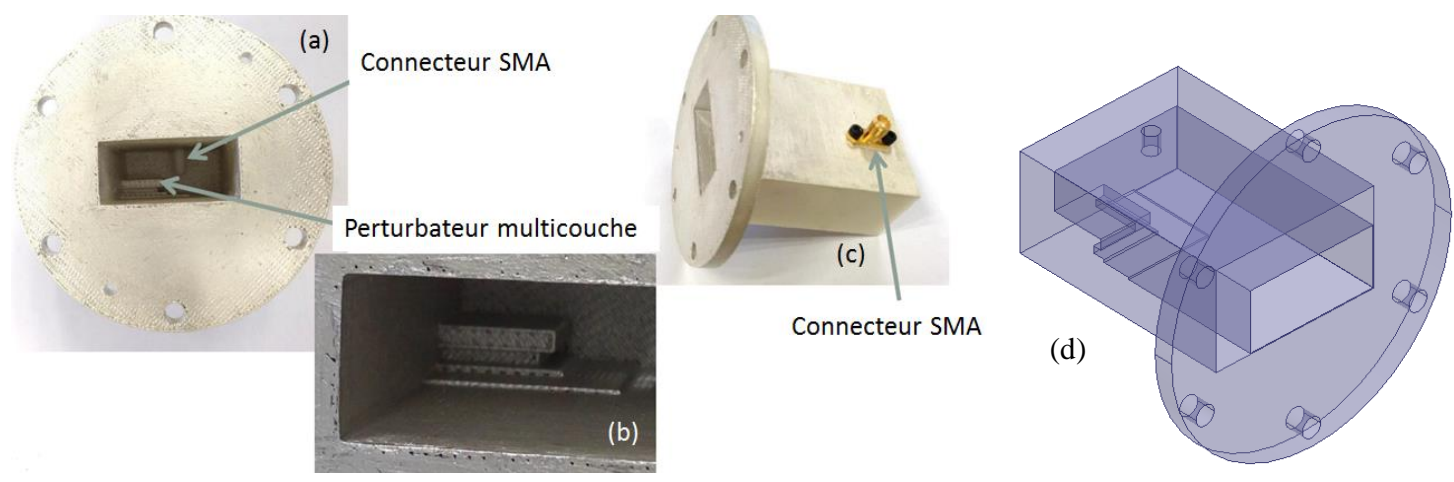

Fig.1. a) Dispositif imprimé en ABS b) Perturbateur multicouche c) Excitation par connecteur SMA d) Dessin 3D de la transition (format STL téléchargeable).

Des trous borgnes de fixation ont été percés et taraudés dans la structure autour du trou de passage du coaxial pour fixer le connecteur coaxial SMA (Fig. 3-c). L'âme centrale enrobée de son Teflon, pénètre de $9 \mathrm{~mm}$ dans le guide d'onde. La figure Fig. 1-d montre le dessin 3D de la structure à imprimer avec des parois de $5 \mathrm{~mm}$ et le flasque de connexion UAR 70 dont le fichier CAO au format stl (STereoLithography de 3D Systems) est téléchargeable. 
Les métallisations intérieures et de la bride de connexion ont été réalisées par une laque argent de marque Ferro.

Après connexion à une transition commerciale de même standard (guide et flasque de connectique) au niveau des flasques des guides d'ondes rectangulaires, les paramètres $\mathrm{S}$ ont été mesurés (Fig. 2).

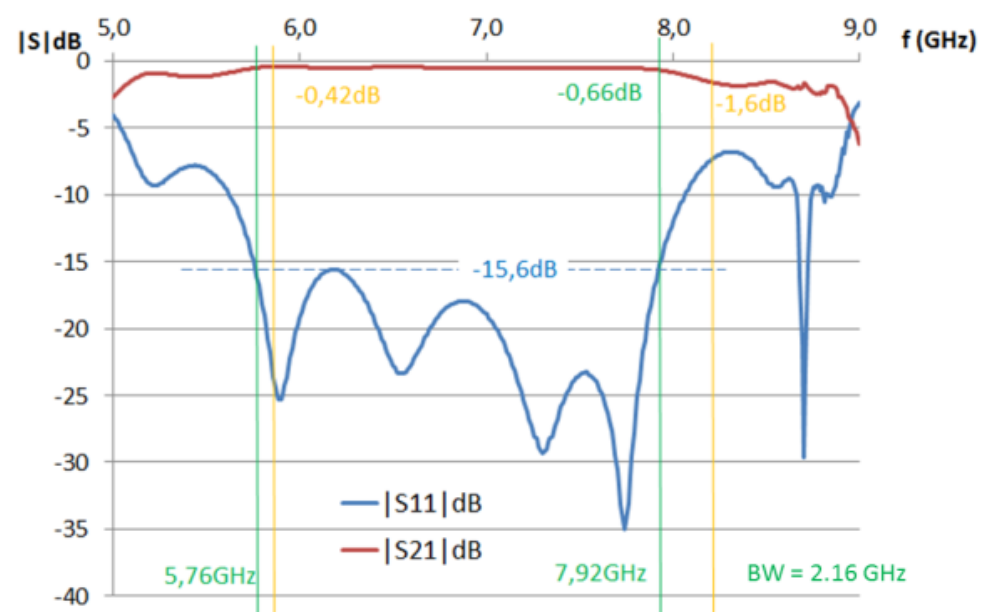

Fig.2. Mesures des paramètres S d'un adaptateur coaxial SMA/WR137

Des pertes d'insertion maximales de $0,66 \mathrm{~dB}$, et une adaptation inférieure à $-15 \mathrm{~dB}$ $(\operatorname{ROS}<1,4)$ ont été obtenues sur une bande de 5,76 à 7,92 GHz. Les résultats sont tout à fait satisfaisants par rapport à ce type de transition coax/WR137. Ce dispositif ne coûte que quelques dizaines d'euros et les temps de conception, de réalisation, de métallisation et de mesures sont de quelques jours. L'impression par technique FDM de dispositifs passifs semble une solution très pertinente dans un contexte pédagogique.

\section{III.Filtre plan-E deux pôles monobloc}

Pour valider la possibilité de monter en fréquence avec la technologie FDM, un filtre deux pôles à inserts métalliques a été conçu pour une bande passante de $200 \mathrm{MHz}$ autour de la fréquence de $10,5 \mathrm{GHz}$ avec une ondulation dans la bande passante de $0,2 \mathrm{~dB}$. Il présente l'avantage d'une synthèse simple (Tchebychev), d'être monobloc et sans dispositif d'accord. Une synthèse de Tchebychev sans pertes avec un logiciel en ligne développé pour ces applications et pour l'enseignement permet de calculer le facteur de qualité extérieur et le couplage k pour les paramètres précédents.

La simulation EM 3D (HFSS) a permis d'obtenir la fréquence de résonance en fonction de l'espace entre les inserts métalliques et les lois de couplage d'entrée/sortie et inter résonateur en fonction des longueurs des inserts (pour une épaisseur fixée à $1 \mathrm{~mm}$ ).

Sur la photo de la figure Fig. 3-a sont représentées les isovaleurs du module du champ électrique qui montrent le confinement du pseudo mode $\mathrm{TE}_{101}$ dans l'espace inter-inserts. La figure Fig. 3-b expose le dispositif 3D à imprimer dont le fichier CAO au format STL est téléchargeable. Il est à noter que le flasque de connectique est au standard UBR 100 associé au standard de guide WR90 et les épaisseurs de matière sont de $5 \mathrm{~mm}$. Sur la figure Fig. 3-c, on distingue les inserts métalliques servant d'iris de couplage qui ont été métallisés comme toute la partie interne du filtre par laque argent. 

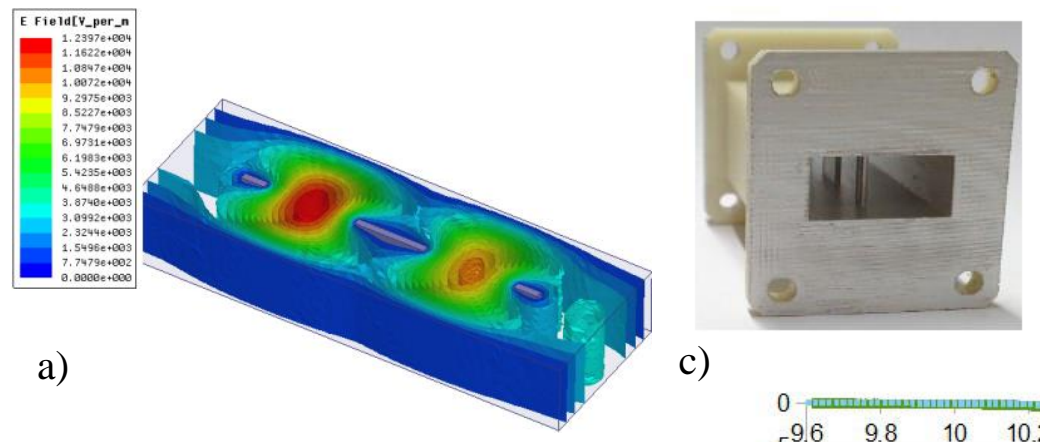

\begin{tabular}{|l|c|c|}
\hline & mesures & simu \\
\hline $\mathbf{S} 21$ max / dB & $-0,5$ & $-0,12$ \\
\hline $\mathbf{F}_{0} / \mathbf{G H z}$ & 10,53 & 10,51 \\
\hline $\mathbf{B}_{\text {peq }} / \mathbf{M H z}$ & 185 & 200 \\
\hline $\mathbf{S 1 1} \mathbf{~} \mathbf{m a x} / \mathbf{d B}$ & $-11,5$ & $-12,45$ \\
\hline $\mathbf{R i p p l e} / \mathbf{d B}$ & 0,2 & 0,23 \\
\hline $\mathbf{T p g} / \mathbf{n s}$ & $>=10$ & $>=1,5$ \\
\hline
\end{tabular}

c)

b)
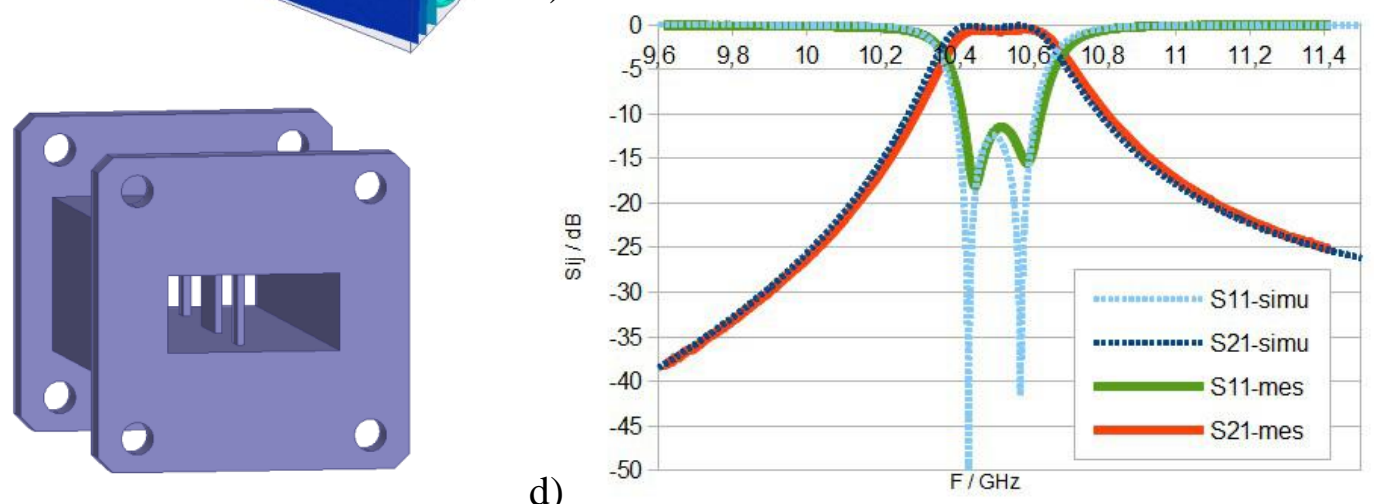

Fig.3. a) Isovaleurs du module du champ électrique dans la structure b) Dessin 3D du filtre (format STL téléchargeable) c) Dispositif imprimé en ABS et peint à la peinture argent, comparaison mesures et simulations d) Comparaison mesures et simulations des $S_{\mathrm{ij}}$ du filtre 2 pôles plan-E.

Les résultats de la figure Fig. 3-d, qui présentent la comparaison entre simulations et mesures du filtre, montrent un bon accord sans post-réglage et démontrent la pertinence du prototypage par impression 3D en technologie FDM dans la bande X. Ces résultats confirment l'intérêt majeur de cette technologie pour l'enseignement des microondes avec un dépassement des limites habituelles des concepts imaginables en projet en termes de qualité, de délais de réalisation et de coût.

\section{Concept de filtre modulaire}

La conception d'un filtre modulaire en impression 3D est maintenant présentée. Il a fait l'objet d'un stage en laboratoire de master «microonde » de deux mois. Le principe proposé est que l'étudiant en TP puisse choisir l'ordre d'un filtre passe bande en clipsant les uns aux autres des blocs (cavités résonantes) autant que nécessaire. La conception et les résultats d'un filtre à 3 et 4 pôles de type Tchebychev sont montrés par la suite. Ce filtre doit avoir des performances conformes aux filtres volumiques, être rapidement et aisément assemblable, de réglage et de maintenance facile et finalement de faible coût (5).

\section{Cavité et guide utilisés}

Le but est de concevoir et de fabriquer un filtre modulaire et réglable constitué de blocs élémentaires qui sont assemblés. Un guide d'onde rectangulaire WR75 est utilisé sur son mode fondamental $\mathrm{TE}_{10}$ pour un fonctionnement en fréquence dans la bande 9 $15 \mathrm{GHz}$. Les cavités résonantes fonctionnent quant à elles sur le mode $\mathrm{TE}_{101}$ (6) et sont cubiques ce qui facilitera l'assemblage des blocs.

L'accord en fréquence se fait par l'intermédiaire de vis métalliques ou plastiques placées au centre de la cavité qui viennent perturber le champ EM et modifier ainsi la fréquence de résonance au détriment du facteur de qualité de la cavité. 
Quand la profondeur de vis augmente, la fréquence de résonance diminue ainsi que le facteur de qualité du résonateur comme l'ont montré les simulations EM (HFSS) pour plusieurs diamètres de vis et de matières (Nylon et métal).

Pour éviter les courants de fuite dans les gaps entre les vis métalliques et les taraudages, qui provoquent des pertes par rayonnement (Fig. 7-b), des vis plastiques sont préférées avec un effet de guide d'onde sous coupure dans les trous taraudés, ce qui limitera les pertes par rayonnement. La faible permittivité des vis (comme le Nylon) est suffisante pour faire varier la fréquence de résonance des cavités et ne pas trop atténuer le facteur de qualité lors de la pénétration de la vis.

\section{$\underline{\text { Etude des couplages }}$}

Le réglage du couplage entrée/sortie à travers le facteur de qualité extérieur $\mathrm{Q}_{\mathrm{e}}$ se fera par l'intermédiaire de vis placées au centre des iris inductifs entre le guide d'onde d'excitation et la cavité. Le couplage inter-résonateur $\mathrm{k}$ se fera entre les deux cavités résonantes $(7,8)$. Une synthèse par matrice de couplage (9) avec un logiciel en ligne développé pour ces applications pour l'enseignement et la recherche (Fig. 4) permet de calculer le facteur de qualité extérieur et le couplage $\mathrm{k}$ pour les paramètres suivants : fréquence centrale $12 \mathrm{GHz}$, bande passante à équi-ondulation: $200 \mathrm{MHz}$, ondulation de $0,2 \mathrm{~dB}$ pour 2, 3 ou 4 résonateurs. Les performances électromagnétiques des résonateurs sont prises en compte à travers un facteur de qualité à vide $\mathrm{Q}_{0}$ estimé.

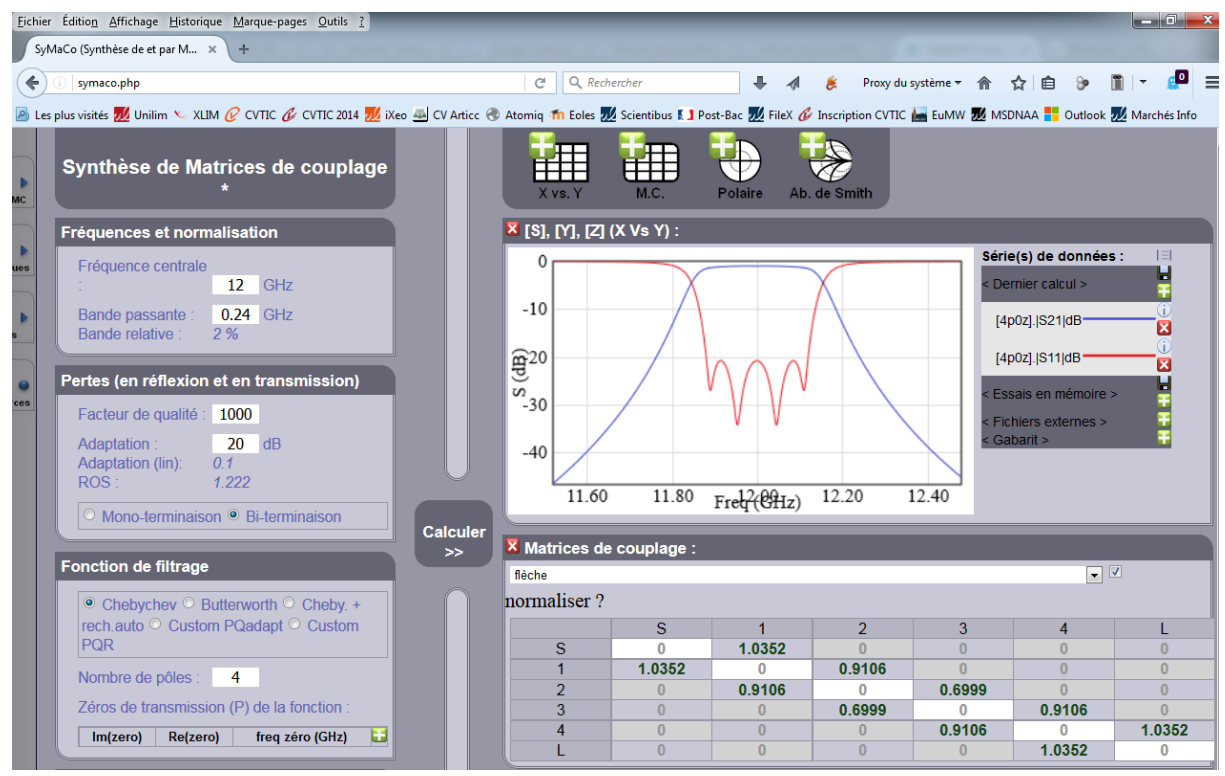

Fig.4. Logiciel en ligne (intranet) de synthèse de filtre par matrice de couplage (9).

Avec le logiciel de synthèse en ligne, les étudiants peuvent changer les différents paramètres du filtre et voir l'impact de ces paramètres sur la réponse du filtre. La figure 5 présente les sélectivités de filtres passe bande de Tchebychev pour 2, 3 et 4 pôles.

Des simulations HFSS sont réalisées en faisant varier les profondeurs de vis dans les iris qui ont tous les mêmes dimensions. Un seul résonateur avec deux guides d'onde d'entrée-sortie est utilisé pour calculer $\mathrm{Q}_{\mathrm{e}}$. 


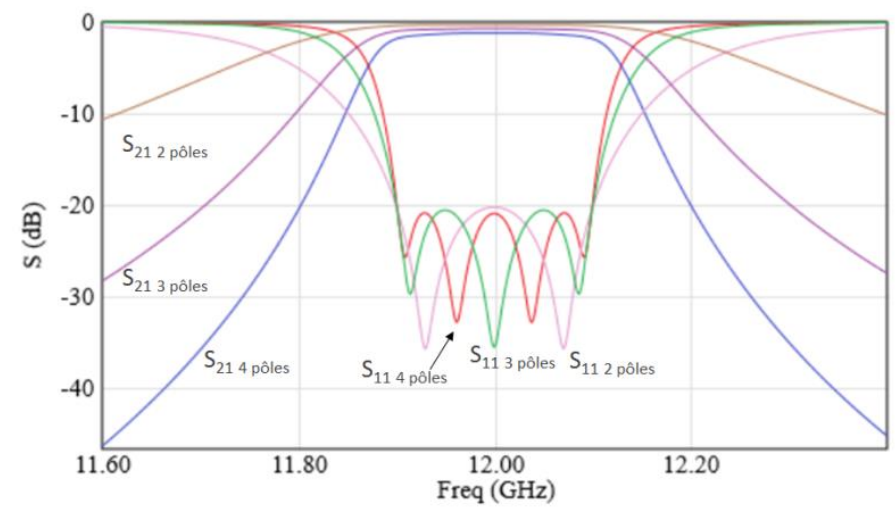

Fig.5. Synthèse de filtre pour 2, 3 et 4 résonateurs ( $f_{0}=12 \mathrm{GHz}, \mathrm{BP}=200 \mathrm{MHz}, \mathrm{S}_{11}=20 \mathrm{~dB}, \mathrm{Q}_{0}=1000$ )

Le couplage inter résonateur $\mathrm{k}$ peut être déterminé par la simulation de deux cavités en faisant varier la profondeur de vis dans l'iris. La figure 6 présente les lois de couplages pour des vis Nylon de $5 \mathrm{~mm}$ de diamètre. Ces valeurs complètent la synthèse du filtre.

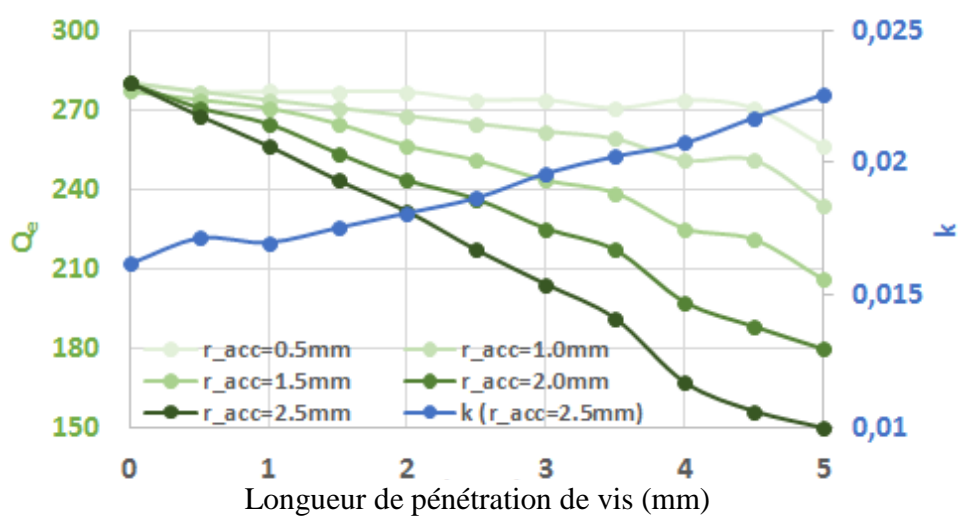

Fig.6. $\mathrm{Q}_{\mathrm{e}}$ et couplage $\mathrm{k}$ en fonction de la profondeur de vis pour différents diamètres de vis.

Ces lois de couplage sont vérifiables par des mesures que peuvent réaliser les étudiants grâce au filtre modulaire en ne conservant qu'un ou deux résonateurs.

\section{Fabrication du filtre modulaire}

L'idée est d'obtenir des blocs simples élémentaires qui peuvent s'assembler et s'aligner afin de créer un filtre passe-bande. Il n'est pas souhaitable d'avoir un système de verrouillage non symétrique et compliqué.

Ainsi, il a été décidé de séparer les fonctions d'alignement des blocs et celles de verrouillage des blocs entre eux.

La fonction d'alignement est réalisée en plaçant sur chaque face à connecter, deux broches et deux trous en opposition (Fig. 7-b). Le verrouillage et plus critique car il peut générer des pertes par courant de fuite si un bon contact entre les blocs n'est pas réalisé.

Le verrouillage des modules se fait par l'insertion de clés de verrouillage dans des trous de forme en négatif des clés (Fig. 7 b).

Plusieurs essais par impression Polyjet ${ }^{\circledR}$ ont été réalisés afin que les pièces s'ajustent idéalement pour un verrouillage efficace mais facilement démontable.

Après cette étape, l'ensemble des parties du filtre modulaire (guide d'onde d'entrée/sortie, résonateurs) a été imprimé (Fig. 7.a, and 7.b). Chaque flasque de jonction possède une gorge pour améliorer le contact électrique. Chaque iris possède un trou taraudé pour permettre l'insertion d'une vis de réglage de couplage. 
Afin de nettoyer des résidus plastiques et les supports des pièces en sortie d'impression, un jet d'eau sous pression est appliqué. Un bain à $20 \%$ de soude à $70{ }^{\circ} \mathrm{C}$ permet de complétement éliminer les résidus difficiles d'accès.

Les dimensions de la structure ont été mesurées est une déviation maximale de $60 \mu \mathrm{m}$ a été observée par rapport aux dimensions nominales.

Les trous ont été percés et taraudés pour des vis de diamètre $5 \mathrm{~mm}$ qui permettent une manipulation aisée à la main et sans outil.

Chaque face en interaction avec le champ EM du dispositif a été métallisée par trois couches successives de peinture à l'argent afin d'avoir un dépôt relativement homogène et suffisamment épais devant la profondeur de peau (Figure 7-a).
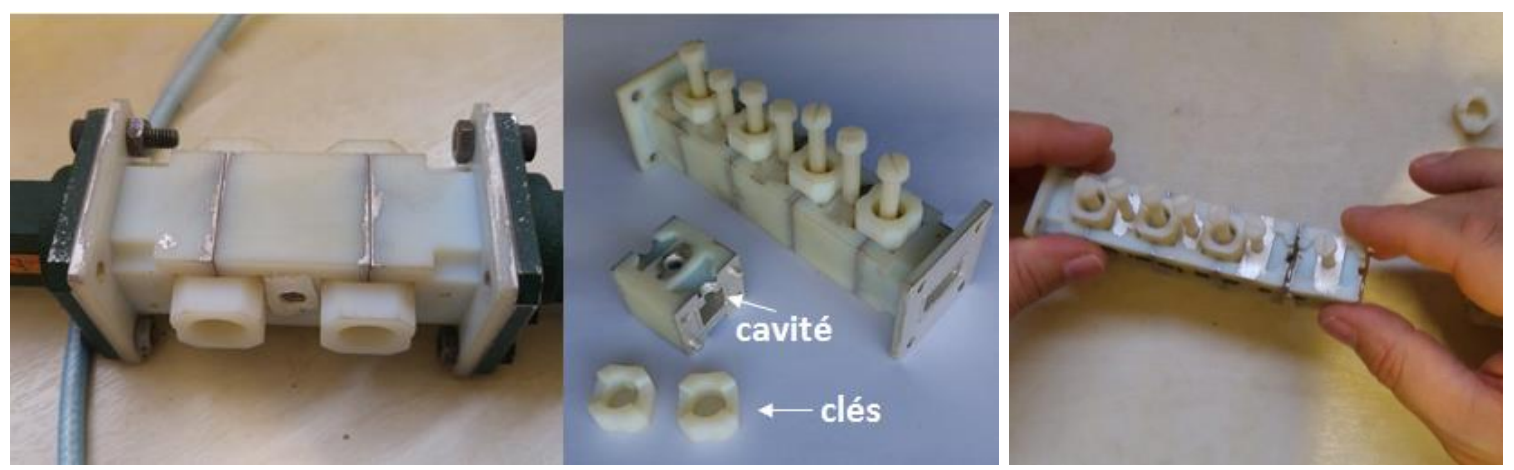

Fig.7. a) Assemblage d'un résonateur, b) 3 résonateurs c) vidéo d'assemblage

Une attention particulière doit être portée sur les trous taraudés pour conserver l'approche modulaire (et des blocs interchangeables) recherchée.

\section{Mesures d'un filtre 3 pôles passe bande}

Les trous ainsi que les taraudages ont été réalisés directement sur le filtre passe-bande 4-poles. Ceci peut être gênant pour l'assemblage avec moins de résonateurs, notamment celui du dernier résonateur assemblé au guide d'excitation qui n'aurait pas le même taraudage d'iris. Pour la prochaine version et pour les iris, tous les taraudages seront faits dans les clés pour permettre un vissage quels que soient les blocs assemblés. Les paramètres $\mathrm{S}$ ont été mesurés après l'étalonnage de l'analyseur de réseaux vectoriel à l'aide d'un kit d'étalonnage WR75. Des vis plastiques de diamètre $5 \mathrm{~mm}$ ont été utilisées pour le réglage facile du dispositif à la main.

Une première mesure d'un seul résonateur (Fig. 7-a) permet de vérifier la variation en fréquence avec la profondeur de la vis dans la cavité (Fig. 8). La fréquence de résonance se décale de $13 \%$ pour toute la longueur de vis dans le guide et le facteur de qualité à vide $\mathrm{Q}_{0}$ décroit de 860 à 140 (84\%).

Ensuite, l'assemblage de 3 résonateurs réalisé (Fig. 7-b), le réglage du filtre avec les spécifications en parenthèse dans le tableau 1, a été effectué dans un temps d'environ 8 minutes. La figure 9 ainsi que le tableau 1 présentent les résultats de mesures. 


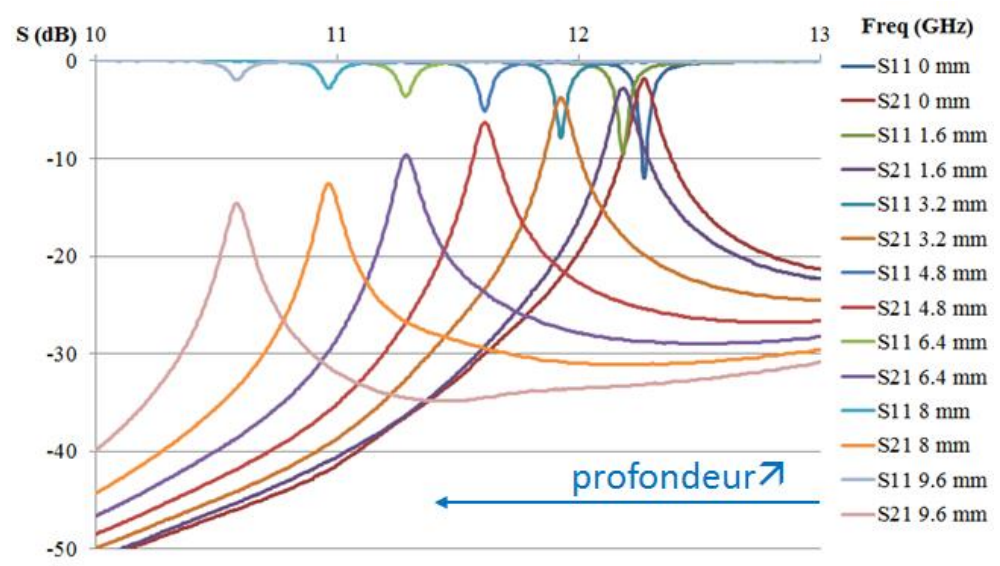

Fig.8. Mesures d'un résonateur en fonction de la longueur de vis dans la cavité

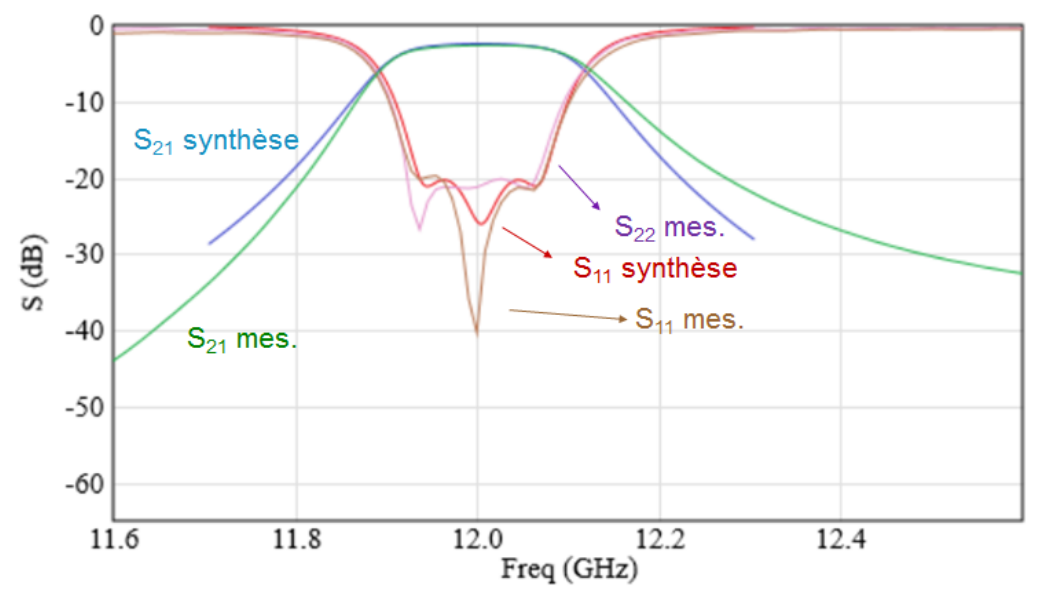

Fig.9. Paramètres $S$, mesures et synthèse d'un filtre 3 pôles.

Un assemblage de 4 résonateurs a été également réglé et les résultats sont comparés avec le gabarit souhaité dans le tableau 1.

Les caractéristiques des deux filtres correspondent avec celles des gabarits souhaités. On observe des pertes d'insertion élevées de $2,7 \mathrm{~dB}$ pour le filtre à 3 résonateurs et de $2,5 \mathrm{~dB}$ pour le filtre à 4 résonateurs.

TABLEAU I. Caractéristiques des filtres mesurés et (gabarits).

\begin{tabular}{cccccc}
\hline Ordre & $\begin{array}{c}\mathbf{f}_{\mathbf{0}} \\
(\mathbf{G H z})\end{array}$ & $\begin{array}{c}\text { Bande passante } \\
(\mathbf{M H z})\end{array}$ & $\begin{array}{c}\text { Adaptation } \\
(\mathbf{d B})\end{array}$ & $\begin{array}{c}\text { Pertes d'insertion } \\
(\mathbf{d B})\end{array}$ & $\mathbf{Q}_{\mathbf{0}}$ extrait \\
\hline 3 & $12,005 \quad(12)$ & $150(150)$ & $19,7(20)$ & 2,7 & 370 \\
4 & $12,09 \quad(12)$ & $235(240)$ & $19,5(20)$ & 2,5 & 400 \\
\hline
\end{tabular}

Ces pertes un peu élevées par rapport aux simulations proviennent d'un ensemble d'éléments qu'il est difficile de distinguer : le Nylon des vis est inconnu en propriétés diélectriques et peut présenter des pertes diélectriques plus importantes que celles escomptées et la qualité de dépôt de la laque argent est difficilement contrôlable à l'intérieur des cavités sans un contrôle destructif. Ces pertes sont néanmoins acceptables pour les objectifs pédagogiques du dispositif. L'adaptation des deux filtres est meilleure que 19,5 dB. Les mesures sont en bon accord avec les courbes issues de la synthèse de filtre de Tchebychev. Les facteurs de qualité des résonateurs des filtres ont été déterminés 
par l'ajustement des courbes simulées grâce à la synthèse par matrice de couplage à celles mesurées (Fig. 4).

\section{Conclusion}

Des dispositifs microondes monobloc à destination de projet d'étudiants ont été réalisés avec succès avec la technique d'impression 3D plastique FDM et les résultats sont en bon accord avec ceux simulés par HFSS sans dispositifs de réglage et ce, jusqu'à $12 \mathrm{GHz}$. La technologie FDM et la métallisation par laque argent des faces permettent d'envisager des dispositifs passifs microondes dont les seules limitations sont les pertes d'insertion et le facteur de qualité de quelques centaines pour les résonateurs liés à la conductivité de la laque argent et des états de surface des parois.

Un filtre modulaire n-pôles passe bande, pour un objectif d'enseignement de la synthèse et réglage de filtre, a été réalisé par impression polyjet ${ }^{@}$.

La technique d'assemblage des blocs élémentaires sans vis rend la manipulation aisée et rapide. Les vis d'accord des fréquences et des couplages en plastique ont montré une bonne capacité d'accord sans trop détériorer les performances du filtre tout en permettant une manipulation simple et sans outil. Une bonne précision de réalisation inférieure à $60 \mu \mathrm{m}$ a été observée pour l'impression polyjet ${ }^{@}$.

Les fonctions d'alignement des blocs et de verrouillage ont été séparées pour une facilité d'utilisation sans perdre en performances de connexion et donc de filtrage.

Les mesures d'un seul résonateur et de filtres 3 et 4 pôles ont été réalisées après peinture à l'argent des surfaces utiles. Le réglage de ces filtres s'est effectué dans des temps compatibles avec une séance de travaux pratiques. Les résultats sont en bon accord avec ceux théoriques. Les outils logiciels en ligne de synthèse de filtre par matrice de couplage améliorent la compréhension des processus de conception et de réglage des filtres des étudiants.

Finalement, ces résultats montrent que le filtre modulaire n-pôles est un moyen efficace d'aborder la conception de filtre, puisque les filtres peuvent être construits avec une réponse spécifique comme un jeu de "Légo technique".

\section{Remerciements}

Les auteurs souhaitent remercier le Pôle Limousin de Microélectronique (PLM) du GIP-CNFM (10) ainsi que l'ANR IDEFI FINMINA (11) pour le co-financement des analyseurs de réseaux vectoriels d'enseignement utilisés pour les mesures.

Sont remerciés également Clément Boudeau, Amel Maati et Jeremy Saucourt, les étudiants de classes préparatoires $2^{\text {ème }}$ année du Lycée Turgot de Limoges et de Master 1

de la formation IXEO de la Faculté des Sciences et Techniques de l'Université de Limoges pour leur travail sur ces projets.

\section{Références}

1. N. Jolly, O. Tantot, N. Delhote, S. Verdeyme, L. Estagerie, L. Carpentier, and D. Pacaud, "Wide range continuously high electrical performance tunable e-plane filter by mechanical translation", In Microwave Conference (EuMC), 44 ${ }^{\text {th }}$ European, pages 351-354, (2014).

2. P. Booth and E. V. Lluch, "Performance enhancement for waveguide filters using additive manufacturing”, In CNES/ESA International Workshop on Micro-wave Filters, 6, (2015).

3. J. Ramón, M. Garai, I. O. Saracho, P. Jorge, A. R. Cruz, J. M. Rebollar, "An Educational Perspective of Low Cost Additive Manufacturing by Fused Filament Fabrication of Microwave Waveguide Passive Devices", Workshop on Additive Manufacturing for RF Passive Hardware, European Microwave Conference, London, United Kingdom, (2016). 
4. J. Sence, W. Feuray, A. Périgaud, O . Tantot, N. Delhote, S. Bila, S. Verdeyme, "Plastic and Metal Additive Manufacturing Technologies for Hyperfrequency Passives Components up to Ka band" in European Microwave Conference, London, United Kingdom, (2016).

5. J. Saucourt, N. Jolly, A. Perigaud, O. Tantot, N. Delhote, S. Bila, S. Verdeyme, R. P Gramond, "Design of 3D Printed Plastic Modular Filters", in European Microwave Conference, London, United Kingdom, (2016).

6. G. Boudouris, “Cavités électromagnétiques", Ed. Dunod, Paris (1971).

7. S. Akatimagool and S. Choocadee, "The development of efficient cwfd simulation tools for waveguide band-pass filter design", In Intelligent Signal Processing and Communications Systems (ISPACS), 2011 International Symposium on, pages 1-4, (2011).

8. Ji-Fuh Liang, H.-C. Chang, and K.A. Zaki, "Design and tolerance analysis of thick iris waveguide bandpass filters", Magnetics, IEEE Transactions on, 29(2):1605-1608, (1993).

9. F. Seyfert and S. Bila, "General synthesis techniques for coupled resonator networks," in IEEE Microwave Magazine, vol. 8, no. 5, pp. 98-104, (2007).

10. GIP-CNFM: Groupement d'Intérêt Public - Coordination Nationale pour la formation en Microélectronique et en nanotechnologies. Website: http://wwww.cnfm.fr

11. IDEFI-FINMINA: Initiative d'Excellence - Formation Innovante en MIcroélectronique et Nanotechnologies, ANR-11-IDFI-0017. Website: http://www.cnfm.fr/VersionFrancaise/actualites/ FINMINA.htm 